\title{
Model of Antenna Modified by Regular Wire Media for Control of its Main Characteristics
}

\author{
Mykola Khobzei, Myhailo Apostolyuk, Dmytro Vovchuk \\ Department of Radio Engineering and Information Security, Yuriy Fedkovych Chernivtsi National University, \\ UKRAINE, Chernivtsi, 2 Kotsubynskyi Str., E-mail: hobze7@gmail.com, mandun1998@gmail.com, \\ dimavovchuk@gmail.com
}

\begin{abstract}
The paper presents the suggestion for the modification of antenna to provide the radiation at the frequencies different from its resonance one. It is possible using wire metamaterials. This approach also allows controlling the resonance frequency and bandwidth.
\end{abstract}

Keywords - wire media, resonance frequency, bandwidth, lattice period, wire radius.

\section{Introduction}

Modern telecommunication and radio devices require new approaches for their development. New systems have a tandency to use microwave and higher frequency ranges. It claims to use novel techniques for the progress of current technologies. Metamaterials are known during long time but it has become the popularity in radio engineering recently. Metamaterial is an electromagnetically homogenous arrangement of artificial structural elements, designed to achieve advantageous and unusual electromagnetic properties $(\varepsilon<0$ and/or $\mu<0)$ [1]. One of the class of artificial materials is a wire media (WM) characterizing by negative value of permittivity. WM consists of the parallel metallic wires with defined length $L$, lattice period $a$ and radius $r$ (Fig. 1). Such materials are usable for a huge number of applications in very wide frequency range from microwave up to optical one [2]. It is possible due to variety methods of their manufacturing and synthesis. Here, the antanna modification by WM are concidered that makes possible to control the resonances and bandwidth.

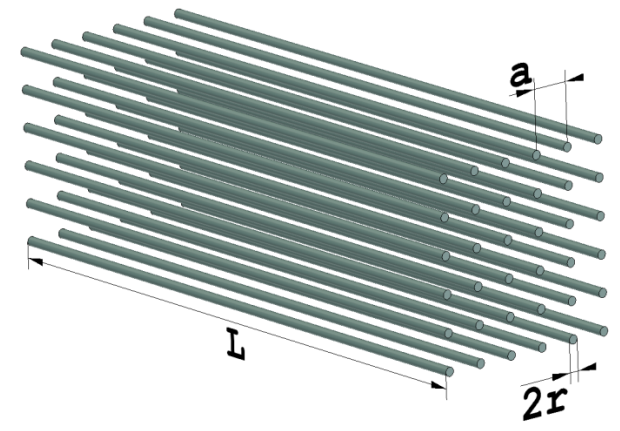

Fig.1. Wire media is an array of metallic wires with length $L$, radius period $2 r$ and lattice period $a$.
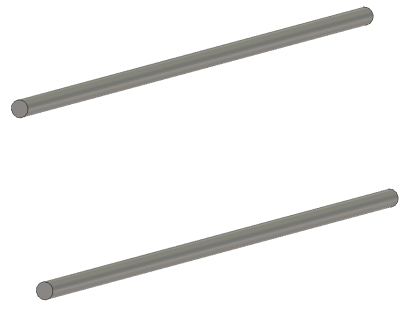

a)

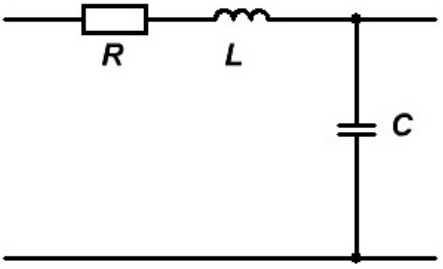

b)

Fig.2. Two-wire transmission line as an elementary cell of WM (a) and equivalent its equivalent RLC circuit model (b).

\section{Main Results}

WM consists of an wire array with fixed finite values of length, lattice period and radius. As an elementary cell of WM is two-wire transmission line. Therefore, the equivalent circuit of such transmission line can be represented by $R L C$-filter for high frequency range. It is known that the general equivalent circuit model of inductance includes ohmic resistance and shunt capacitance. However, in the suggested equivalent model those can be neglected because the wires are straight and concedered as an perfect conductors. Also, the general equivalent circuit model of capacitance includes parallel insulation resistance as well as series resistance and inductance. The equivalent circuit of our elementary cell excudes such components due to the 
insulator is air or vacuum that means the parallel resistance is close to infinity and any ohmic contacts are absent.

The equivalent circuit model demonstrates the possibility to control by transmission and radiation characteristics by changing of WM parameters. These principle was exploited for modification of antenna with controllable values resonance and bandwidth. The experimental setup consists of waveguide port with inductive loaded aperture (antenna with poor radiation into free space) and embedded WM sample (Fig. 3). For investigation two different WM samples were manufactured with values of the wires radii and lattice periods $0.5 \mathrm{~mm}$ and $10 \mathrm{~mm}$ as well as $0.75 \mathrm{~mm}$ and $6 \mathrm{~mm}$ respectively. The overlapping between port aperture and WM was changing for each WM that shows any impacts on radiation. However, one can see that an increasing of wire radius leads to increasing of the value of resonance frequency 1.02 and $1.275 \mathrm{GHz}$ and decreasing of lattice period leads to increasing of the value of bandwidth up to $80 \mathrm{MHz}$ (Fig. 4). It shows a strong dependence between the main parameters of WM and radiation properties of the modified antenna.



Fig.3. Experimental setup for investigation of modified antenna's properties.

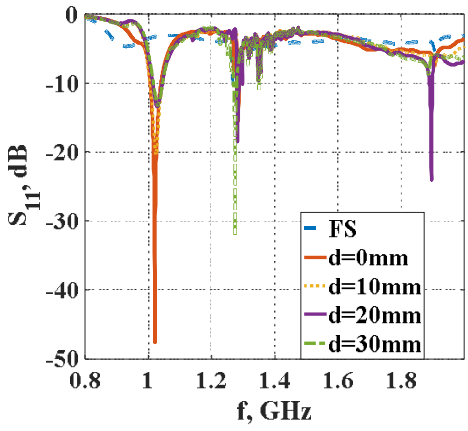

a)



b)

Fig.4. $\mathrm{S}_{11}$ dispersion of modified antenna by $\mathrm{WM}$ for $0.5 \mathrm{~mm}$ and $10 \mathrm{~mm}$ (a) as well as $0.75 \mathrm{~mm}$ and $6 \mathrm{~mm}$ (b) of the wires radii and lattice periods, respectively.

\section{Conclusion}

In this paper the antenna modified by the metastructure that consists of parallel metallic wires was suggested. Such modification allows to radiate electromagnetic waves at the frequencies which do not correspond the resonance frequencies of antenna for the radiation into the free space. For the investigation two WM samples with different values of wires radii and lattice period were constructed in accordance with the equivalent circuit model. It gives a possibility to define the strong dependence between the main parameters of WM and radiation properties of modified antenna. The obtained results can be used for development of antennas when the control of resonance frequency and bandwidh are required.

\section{References}

[1] Sihvola A., "Metamaterials in electromagnetic," Physical Review Letters, Oct. 2000, Vol.85, №18, pp. 3966-3069.

[2] Simovski C. R. et al., "Wire metamaterials: Physics and application," Adv. Mater.- 2012, Vol. 24, pp. 4229-4248. 